\title{
Flow and heat transfer of a third grade fluid past an exponentially stretching sheet with partial slip boundary condition
}

\author{
Bikash Sahoo \\ Department of Mathematics, \\ National Institute of Technology, Rourkela, Rourkela-769008, Orissa, \\ INDIA, Ph: +916612462706 \\ Sébastien Poncet \\ Laboratoire M2P2 UMR 6181 CNRS - Aix-Marseille Université, Marseille, \\ FRANCE - Tel. +33491118549
}

\begin{abstract}
Non-Newtonian boundary layer flow and heat transfer over an exponentially stretching sheet with partial slip boundary condition has been studied in this paper. The flow is subject to a uniform transverse magnetic field. The heat transfer analysis has been carried out for two heating processes, namely (i) with prescribed surface temperature (PST), and (ii) prescribed heat flux (PHF). Suitable similarity transformations are used to reduce the resulting highly nonlinear partial differential equation into ordinary differential equation. An effective second order numerical scheme has been adopted to solve the obtained differential equations. The important finding in this communication is the combined effects of the partial slip and the third grade fluid parameters on the velocity, skin-friction coefficient and the temperature boundary layer. It is found that the third grade fluid parameter $\beta$ increases the momentum boundary layer thickness and decreases the thermal boundary layer thickness.
\end{abstract}

Key words: Third grade fluid, Partial slip, Magnetic field, Heat transfer, Finite difference method.

PACS: 47.11.Bc, 47.15.Cb, 47.50.Cd, 47.65.Cb

Email addresses: bikashsahoo@nitrkl.ac.in (Bikash Sahoo), poncet@l3m.univ-mrs.fr (Sébastien Poncet). 


\section{Introduction}

The study of laminar boundary layer flow over a stretching sheet has received considerable attention in the past due to its applications in the industries, for example, materials manufactured by extrusion process, the boundary layer along a liquid film in condensation process and the heat treated materials traveling between a feed roll and the wind-up roll or on conveyor belt poses the features of a moving continuous surface. The flow and heat transfer phenomena over stretching surface have promising applications in a number of technological processes including production of polymer films or thin sheets. The no-slip boundary condition (the assumption that a liquid adheres to a solid boundary) is one of the central tenets of the Navier-Stokes theory. However, there are situations wherein this condition does not hold. Partial velocity slip may occur on the stretching boundary when the fluid is particulate such as emulsions, suspensions, foams and polymer solutions. Navier [1] proposed a slip boundary condition wherein the slip depends linearly on the shear stress. The inadequacy of the no-slip condition is evident for most non-Newtonian fluids. One can refer the works of Andersson [2], Sahoo [3,4], Sahoo and Do [5], Ariel [6], Sajid etal [7] and all the references therein regarding the flow and heat transfer of Newtonian and different non-Newtonian fluids past stretching sheets with slip and no-slip boundary conditions. Further the effects of slip and non-Newtonian flow parameters on the boundary layer flows can be seen in $[8-10]$.

Elbashbeshy [11] has added a new dimension in his investigation by considering the flow and heat transfer of a Newtonian fluid over an exponentially stretching continuous surface. He considered an exponential similarity variable and exponential stretching velocity distribution on the coordinate considered in the direction of stretching. Partha et al [12] have discussed the effects of viscous dissipation on the mixed convection heat transfer from an exponentially stretching surface in a quiescent viscous fluid. Sajid and Hayat [13] have investigated the influence of thermal radiation on the boundary layer flow and heat transfer of an incompressible viscous fluid due to an exponentially stretching sheet. Recently, Pal [14] has carried out an analysis to describe mixed convection heat transfer in the boundary layers on an exponentially stretching continuous surface with an exponential temperature distribution in the presence of magnetic field, viscous dissipation and internal heat generation/absorption. Further one can refer the works of Al-Odat et. al. [15] and Bidin and Nazar [16] regarding the flow and heat transfer of viscous fluid past an exponential stretching sheet. Khan and Sanjayanand [17], and Khan [18] have extended the work of Elbashbeshy [11] to the flow and heat transfer of a thermodynamically compatible second grade fluid. In a subsequent study (see Ref. [19]), they have added the mass transfer aspect. 
In this work, the steady laminar flow and heat transfer of an electrically conducting third grade fluid over an exponentially stretching sheet with partial slip is considered. To the best of our knowledge, no attention has been given to the combined effects of partial slip and the magnetic field on the boundary layer flow and heat transfer of a third grade fluid past an exponentially stretching sheet.

\section{Formulation of the problem}

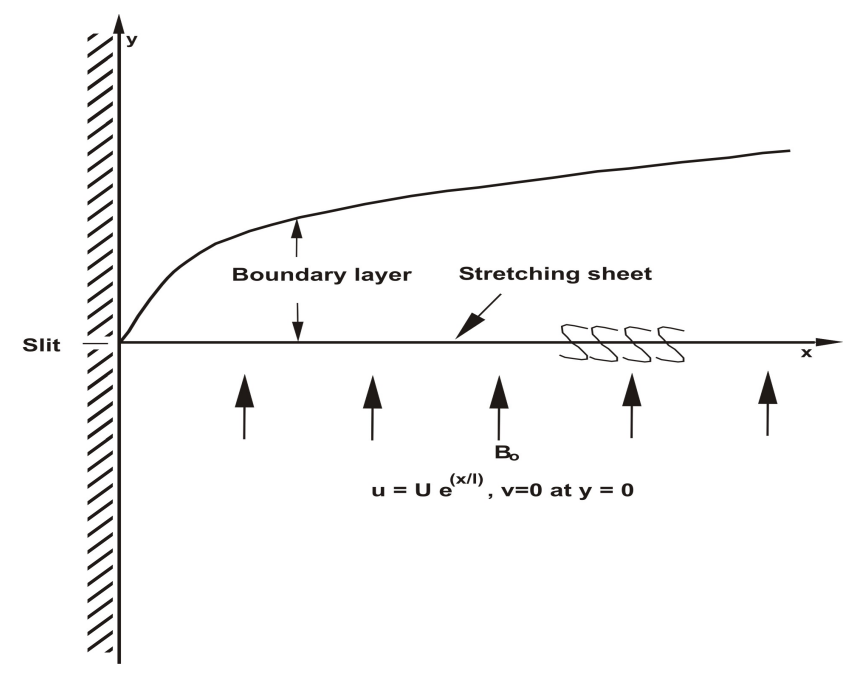

Fig. 1. Sketch of the flow past an exponentially stretching sheet.

We consider the two-dimensional steady-state boundary layer flow and heat transfer of an incompressible, electrically conducting fluid of third grade over a stretching sheet. The constitutive equation of the thermodynamically compatible third grade fluid is given by [20]

$$
\mathbf{T}=-p \mathbf{I}+\mu \mathbf{A}_{1}+\alpha_{1} \mathbf{A}_{2}+\alpha_{2} \mathbf{A}_{1}^{2}+\beta_{3}\left(\operatorname{tr} \mathbf{A}_{1}^{2}\right) \mathbf{A}_{1}
$$

where $-p$ is the pressure, $\alpha_{1}$ and $\alpha_{2}$ are the normal stresses and $\beta_{3}$ is the material constant. $\mathbf{A}_{1}$ and $\mathbf{A}_{2}$ are the first two Rivlin-Ericksen tensors.

The sheet is coinciding with the plane $y=0$ (see Fig. 1). The flow is assumed to be generated by stretching of the elastic boundary sheet from a slit with a large force such that the velocity of the boundary sheet is an exponential order of the flow directional coordinate $x$. The flow takes place in the upper half plane $y>0$. A uniform magnetic field $\mathbf{B}=\left(0, B_{0}, 0\right)$ is imposed along the $y$-axis. 


\section{Flow Analysis}

For the physical problem, where the stretching of the boundary surface is assumed to be such that the flow directional velocity is of exponential order of the flow directional coordinate, the conventional no-slip boundary conditions are (see Ref. [11,17,21]):

$$
\begin{aligned}
& u=U_{w}(x)=U_{0} \exp \left(\frac{x}{l}\right), \quad v=0 \quad \text { at } y=0, \\
& u=0, \quad \text { as } y \rightarrow \infty
\end{aligned}
$$

Here $U_{0}$ is the reference velocity and $l$ is the reference length. The above exponential boundary condition is valid only when $x \ll l$, which occurs very near to the slit.

Making the usual boundary layer approximations for the non-Newtonian third grade fluid (see Ref. [22]), the equations of continuity and motion can be written as

$$
\begin{gathered}
\frac{\partial u}{\partial x}+\frac{\partial v}{\partial y}=0 \\
u \frac{\partial u}{\partial x}+v \frac{\partial u}{\partial y}=\nu \frac{\partial^{2} u}{\partial y^{2}}+\frac{\alpha_{1}}{\rho}\left[u \frac{\partial^{3} u}{\partial x \partial y^{2}}+\frac{\partial u}{\partial x} \frac{\partial^{2} u}{\partial y^{2}}+3 \frac{\partial u}{\partial y} \frac{\partial^{2} v}{\partial y^{2}}+v \frac{\partial^{3} u}{\partial y^{3}}\right] \\
+\frac{2 \alpha_{2}}{\rho} \frac{\partial u}{\partial y} \frac{\partial^{2} v}{\partial y^{2}}+\frac{6 \beta_{3}}{\rho}\left(\frac{\partial u}{\partial y}\right)^{2} \frac{\partial^{2} u}{\partial y^{2}}-\frac{\sigma B_{0}^{2}}{\rho} u .
\end{gathered}
$$

The appropriate Navier's slip boundary conditions [1] of the velocity field are

$u-U_{0} \exp \left(\frac{x}{l}\right)=\lambda_{1} \nu\left[\frac{\partial u}{\partial y}+\frac{\alpha_{1}}{\mu}\left(2 \frac{\partial u}{\partial x} \frac{\partial u}{\partial y}+v \frac{\partial^{2} u}{\partial y^{2}}+u \frac{\partial^{2} u}{\partial x \partial y}\right)+2 \frac{\beta_{3}}{\mu}\left(\frac{\partial u}{\partial y}\right)^{3}\right]$, at $y=0$

$v(0)=0, \quad u \rightarrow 0 \quad$ as $\quad y \rightarrow \infty$.

Eqn. (4) can be rewritten in terms of a stream function $\psi(x, y)$ such that the continuity equation (3) is automatically satisfied. Hence

$$
u=\frac{\partial \psi}{\partial y} \quad v=-\frac{\partial \psi}{\partial x}
$$

We choose the stream function to be

$$
\psi(x, y)=\sqrt{2 \nu l U_{0}} \varphi(\zeta) \exp \left(\frac{x}{2 l}\right),
$$

where

$$
\zeta=y \sqrt{\frac{U_{0}}{2 \nu l}} \exp \left(\frac{x}{2 l}\right) .
$$


With the help of Eqns. (6)-(8), Eqn. (4) gets reduced to,

$\varphi^{\prime \prime \prime}-2 \varphi^{\prime 2}+\varphi \varphi^{\prime \prime}+K\left[3 \varphi^{\prime} \varphi^{\prime \prime \prime}-\frac{1}{2} \varphi \varphi^{i v}\right]-\left(\frac{9}{2} K+3 L\right) \varphi^{\prime 2}+3 \beta \varphi^{\prime \prime \prime} \varphi^{\prime \prime 2}-2 M_{n} \varphi^{\prime}=0$,

where $K=\frac{\alpha_{1} U_{0}}{\rho \nu l}, L=\frac{\alpha_{2} U_{0}}{\rho \nu l}, \beta=\frac{\beta_{3} U_{0}^{3}}{\rho \nu^{2} l}, M_{n}=\frac{\sigma B_{0}^{2} l}{\rho U_{0}}$ are the non-dimensional viscoelastic parameter, cross-viscous parameter, the third grade fluid parameter and the magnetic interaction parameter respectively.

The corresponding partial slip boundary conditions (5) on $\varphi$ become

$$
\begin{aligned}
& \varphi(0)=0, \quad \varphi^{\prime}(0)-1=\lambda \varphi^{\prime \prime}(0)\left[1+\frac{7}{2} K \varphi^{\prime}(0)+\beta \varphi^{\prime \prime 2}(0)\right], \\
& \varphi^{\prime}(\zeta) \rightarrow 0 \quad \text { as } \quad \zeta \rightarrow \infty
\end{aligned}
$$

where the slip factor, $\lambda=\lambda_{1} \sqrt{\frac{U_{0}}{2 \nu l}}$ represents the relative importance of the slip to viscous effects.

\subsection{Heat transfer analysis}

The thermal boundary layer equation for the thermodynamically compatible third grade fluid with viscous dissipation, work done due to deformation and Joule heating is

$\rho c_{p}\left(u \frac{\partial T}{\partial x}+v \frac{\partial T}{\partial y}\right)=\kappa \frac{\partial^{2} T}{\partial y^{2}}+\mu\left(\frac{\partial u}{\partial y}\right)^{2}+\alpha_{1} \frac{\partial u}{\partial y} \frac{\partial}{\partial y}\left(u \frac{\partial u}{\partial x}+v \frac{\partial v}{\partial y}\right)+2 \beta_{3}\left(\frac{\partial u}{\partial y}\right)^{4}+\sigma B_{0}^{2} u^{2}$

where $c_{p}$ is the specific heat at constant pressure, $\kappa$ is the thermal conductivity, $T$ is the temperature, and $T_{\infty}$ is the temperature of the ambient fluid. In order to solve the above energy equation, we consider the following two general cases of non-isothermal temperature boundary conditions:

\subsubsection{Prescribed exponential order surface temperature (PST case)}

The thermal boundary conditions for the energy equation in PST case are,

$$
\begin{aligned}
& T=T_{w}=T_{\infty}+T_{0} \exp \left(\frac{\nu_{0} x}{2 l}\right) \quad \text { at } y=0, \\
& T=T_{\infty} \quad \text { as } y \rightarrow \infty
\end{aligned}
$$

where $\nu_{0}, T_{0}$ are the parameters of temperature distribution on the stretching surface, and $T_{\infty}$ is the temperature far away from the stretching sheet.

In order to obtain the similarity solution for the temperature, we define the 
dimensionless temperature variable $\theta$ as,

$$
\theta(\zeta)=\frac{T-T_{\infty}}{T_{w}-T_{\infty}}
$$

With this, the energy equation (11) takes the following non-dimensional form:

$$
\theta^{\prime \prime}+P_{r} \varphi \theta^{\prime}-P_{r} \nu_{0} \varphi^{\prime} \theta=-P_{r} E_{c}\left[\varphi^{\prime \prime 2}+\frac{K}{2} \varphi^{\prime \prime}\left(\varphi^{\prime} \varphi^{\prime \prime}-\varphi \varphi^{\prime \prime \prime}\right)+2 \beta \varphi^{\prime \prime 4}+M_{n} \varphi^{\prime 2}\right],
$$

where $P_{r}=\frac{\mu c_{p}}{\kappa}$ is the Prandtl number and $E_{c}=\frac{U_{0}^{2}}{c_{p} T_{0}}\left(\frac{U_{w}}{U_{0}}\right)^{\frac{4-\nu_{0}}{2}}$ is the Eckert number. The corresponding thermal boundary conditions are

$$
\begin{aligned}
& \theta=1 \quad \text { at } \zeta=0, \\
& \theta \rightarrow 0 \quad \text { as } \zeta \rightarrow \infty
\end{aligned}
$$

\subsubsection{Prescribed exponential order power law heat flux (PHF case)}

For this case, we employ the following prescribed exponential law heat flux boundary conditions:

$$
\begin{aligned}
-\kappa\left(\frac{\partial T}{\partial y}\right)= & T_{1} \exp \left(\frac{\nu_{1}+1}{2 l}\right) x \quad \text { at } y=0, \\
T \rightarrow T_{\infty} & \text { as } y \rightarrow \infty
\end{aligned}
$$

where $\nu_{1}$ and $T_{1}$ are the parameters of temperature distribution on the stretching surface. We define the dimensionless temperature $g(\zeta)$ in the $P H F$ case as,

$$
g(\zeta)=\frac{T-T_{\infty}}{\frac{T_{1}}{\kappa} \sqrt{\frac{2 \nu l}{U_{0}}} \exp \left(\frac{\nu_{1} x}{2 l}\right)} .
$$

With this dimensionless variable, and Eqns. (6)-(8), the energy boundary layer equation (11) takes the form

$$
g^{\prime \prime}+P_{r} \varphi g^{\prime}-\operatorname{Pr} \nu_{1} \varphi^{\prime} g=-P_{r} E_{c}\left[\varphi^{\prime 2}+\frac{K}{2} \varphi^{\prime \prime}\left(\varphi^{\prime} \varphi^{\prime \prime}-\varphi \varphi^{\prime \prime \prime}\right)+2 \beta \varphi^{\prime \prime 4}+M_{n} \varphi^{\prime 2}\right]
$$

where

$$
E_{c}=\frac{U_{0}^{2} \kappa}{c_{p} T_{1} \sqrt{\frac{2 \nu l}{U_{0}}}}\left(\frac{U_{w}}{U_{0}}\right)^{\frac{4-\nu_{1}}{2}},
$$

which is different from the Eckert number in the PST case, and all other parameters are the same as before. The corresponding thermal boundary conditions are

$$
\begin{aligned}
& g^{\prime}=-1 \quad \text { at } \zeta=0, \\
& g \rightarrow 0 \quad \text { at } \zeta \rightarrow \infty
\end{aligned}
$$




\section{Numerical solution of the problem}

The system of non-linear Eqns. (9), (14) and (9), (18) are solved under the boundary conditions (10), (15) and (10), (19) respectively. It is evident that the order of each system of equations exceeds the number of available adherence boundary conditions. Hence, the solution of the differential equations can not proceed numerically using any standard integration routine. Based on the governing equation, $\varphi^{\prime \prime \prime}(0)$ is related to $\varphi^{\prime}(0)$ and $\varphi^{\prime \prime}(0)$ as

$$
\varphi^{\prime \prime \prime}(0)=\frac{2 \varphi^{\prime 2}(0)+\left(\frac{9}{2} K+3 L\right) \varphi^{\prime \prime 2}(0)+2 M_{n} \varphi^{\prime}(0)}{1+3 K \varphi^{\prime}(0)+3 \beta \varphi^{\prime \prime 2}(0)}
$$

It is clear that if $\varphi^{\prime}(0), \varphi^{\prime \prime}(0)$ and $\theta^{\prime}(0)(g(0)$ for $P H F$ case) are known, then all the successive derivatives of $\varphi$ and $\theta$ at $\zeta=0$ can be determined. Hence a Taylor series expansion can be developed for $\varphi(\zeta)$ and $\theta(\zeta)$ in terms of $\varphi^{\prime}(0), \varphi^{\prime \prime}(0)$ and $\theta^{\prime}(0)$ and consequently we do not require any extra boundary conditions. However, it is possible to use Taylor series expansion to obtain the solution of the above systems of equations up to some small value of $\zeta$, say $\zeta_{c}$, and then the solution can be further carried out by using a suitable integration routine such as the Runge-Kutta method. But in practice, if one employs the Taylor series expansion to obtain the solution in $0 \leq \zeta \leq \zeta_{c}$, a large number of terms will be needed to match the accuracy demanded by RungeKutta method. This can become quite unwieldy for the present complicated problem with partial slip boundary conditions. It appears that the attempts of integrating the above system of nonlinear Eqns. (9) and (14) (or (9) and (18)) subject to the relevant slip boundary conditions by forward integration relying solely on the initial conditions on $\varphi, \varphi^{\prime}, \varphi^{\prime \prime}$ and $\theta^{\prime}$ (or $g$ ) are unable to produce the solution of the system. Ultimately, in view of these drawbacks in the aforementioned routine algorithms, and our experimentations of integrating such systems of equations with inadequate boundary conditions, we deemed it prudent to try a similar second order numerical scheme as described in the previous investigation [3-5].

The semi-infinite domain of integration $\zeta \in[0, \infty)$ is replaced by a finite domain $\zeta \in\left[0, \zeta_{\infty}\right)$, where $\zeta_{\infty}$ is sufficiently large. To explain the solution scheme developed in this study, we start first by introducing the following variables:

$$
y_{1}=\varphi, \quad y_{2}=\varphi^{\prime}, \quad y_{3}=\varphi^{\prime \prime}, \quad y_{4}=\theta(=g \text { for PHF case })
$$


Now the system of equations (9), (14) [or (9), (18)] can be written as ${ }^{1}$

$$
\begin{aligned}
y_{3}^{\prime}-2 y_{2}^{2}+y_{1} y_{3}+K\left[3 y_{2} y_{3}^{\prime}-\frac{1}{2} y_{1} y_{3}^{\prime \prime}\right]-\left(\frac{9}{2} K+3 L\right) y_{3}^{2}+3 \beta y_{3}^{\prime} y_{3}^{2}-2 M_{n} y_{2}=0, \\
y_{4}^{\prime \prime}+P_{r} y_{1} y_{4}^{\prime}-P_{r} \nu_{0} y_{2} y_{4}+P_{r} E_{c}\left[y_{3}^{2}+\frac{K}{2} y_{3}\left(y_{2} y_{3}-y_{1} y_{3}^{\prime}\right)+2 \beta y_{3}^{4}+M_{n} y_{2}^{2}\right]=0, \\
y_{2}^{\prime}=y_{3}, \\
y_{1}^{\prime}=y_{2} .
\end{aligned}
$$

The boundary conditions in terms of the new variables are,

$$
\begin{gathered}
y_{1}(0)=0, \quad y_{2}(0)-1=\lambda y_{3}(0)\left[1+\frac{7}{2} K y_{2}(0)+\beta y_{3}^{2}(0)\right], \quad y_{2}\left(\zeta_{\infty}\right) \rightarrow 0, \\
y_{4}(0)=1, \quad y_{4}\left(\zeta_{\infty}\right) \rightarrow 0, \quad \text { PST case } \\
y_{4}^{\prime}(0)=-1, \quad y_{4}\left(\zeta_{\infty}\right) \rightarrow 0 . \quad \text { PHF case }
\end{gathered}
$$

Eqns.(22)-(25) are discretized using finite difference approximations. Setting up the mesh

$$
\zeta_{i}=i h \quad(i=0,1,2, \ldots, n),
$$

where $h$ is the mesh-size and $n$ is a suitably large integer, so that $\zeta_{\infty}$, the numerical infinity can be reasonably approximated by $n h$; and replacing the derivatives in Eqns. (22) and (23) by the usual central difference formulae, we obtain

$$
\begin{aligned}
\frac{y_{3}^{j+1}-y_{3}^{j-1}}{2 h}-2\left(y_{2}^{j}\right)^{2} & +y_{1}^{j} y_{3}^{j}+K\left[3 y_{2}^{j}\left(\frac{y_{3}^{j+1}-y_{3}^{j-1}}{2 h}\right)-\frac{1}{2} y_{1}^{j}\left(\frac{y_{3}^{j+1}-2 y_{3}^{j}+y_{3}^{j-1}}{h^{2}}\right)\right] \\
& -\left(\frac{9}{2} K+3 L\right)\left(y_{3}^{j}\right)^{2}+3 \beta\left(\frac{y_{3}^{j+1}-y_{3}^{j-1}}{2 h}\right)\left(y_{3}^{j}\right)^{2}-2 M_{n} y_{2}^{j}=0
\end{aligned}
$$

$$
\begin{aligned}
\frac{y_{4}^{j+1}-2 y_{4}^{j}+y_{4}^{j-1}}{h^{2}} & +P_{r} y_{1}^{j}\left(\frac{y_{4}^{j+1}-y_{4}^{j-1}}{2 h}\right)-P_{r} \nu_{0} y_{2}^{j} y_{4}^{j}+P_{r} E_{c}\left[\left(y_{3}^{j}\right)^{2}\right. \\
& \left.+\frac{K}{2} y_{3}^{j}\left\{y_{2}^{j} y_{3}^{j}-y_{1}^{j}\left(\frac{y_{3}^{j+1}-y_{3}^{j-1}}{2 h}\right)\right\}+2 \beta\left(y_{3}^{j}\right)^{4}+M_{n}\left(y_{2}^{j}\right)^{2}\right]=0 .
\end{aligned}
$$

In Eqns. (24) and (25) the derivatives are replaced by the central difference quotients centered at the point $i+\frac{1}{2}$, and the right hand side is replaced by the average of the values of $y$ at the mesh points $i$ and $i+1$. Thus, the Eqns. (24)

$\overline{1 \text { Read }} \nu_{1}$ for PHF case. 
and (25) are discretized as

$$
\begin{aligned}
& \frac{y_{2}^{j+1}-y_{2}^{j}}{h}=\frac{1}{2}\left(y_{3}^{j}+y_{3}^{j+1}\right), \\
& \frac{y_{1}^{j+1}-y_{1}^{j}}{h}=\frac{1}{2}\left(y_{2}^{j}+y_{2}^{j+1}\right) .
\end{aligned}
$$

It is clear that the approximations introduced in discretization have an order $O\left(h^{2}\right)$. Eqns. (29) to (32) can be solved for $y_{3}^{j+1}, y_{4}^{j+1}, y_{2}^{j+1}$ and $y_{1}^{j+1}$ respectively.

Eqns. (31) and (32) are two-term recurrence relations, whereas Eqns. (29) and (30) are three-term recurrence relations at mesh points $i-1, i, i+1$. Thus, in order to start the recursion in Eqns. (29) and (30) two starting values are required, in contrast to one for each of the Eqns. (31) and (32). However, as pointed out earlier, if $\varphi^{\prime}(0), \varphi^{\prime \prime}(0)$ and $\theta^{\prime}(0)(g(0)$ for $P H F$ case) are known, then the Taylor series expansion can be utilized to calculate the values of $\varphi^{\prime \prime}(h)$ and $\theta(h)$, i.e. $y_{3}^{1}$ and $y_{4}^{1}$ respectively. The integration now proceeds as described in our previous works. The only remaining problem is to find the appropriate values of $\varphi^{\prime}(0), \varphi^{\prime \prime}(0)$ and $\theta^{\prime}(0)(g(0)$ for $P H F$ case), such that the terminal boundary conditions in (26) and (27) are satisfied. Thus, we have at our hand a three parameter zero-finding problem for which we have used the effective Broyden's method [23,24]. This method is quite efficient in the sense that it avoids the calculation of the Jacobian matrix (contrary to Newtons method) by obtaining approximations to them involving only function values. The use of Broydens method has helped us in achieving the greatest accuracy with the least computational cost.

The Richardson's extrapolation is used to hike the accuracy of the algorithm to $O\left(h^{4}\right)$. In fact, this scheme is used to obtain highly accurate results by combining the computed values obtained by using a certain method with different step sizes. In general the value of $\zeta_{\infty}$ should be varied depending on the values of $K, L$ and $\beta$. The iterations for locating the missing initial conditions were continued till an accuracy of $O\left(10^{-6}\right)$ was attained. With a reasonably proper choice of the initial guesses, it required $9-11$ iterations to attain the above accuracy.

\section{$5 \quad$ Results and discussions}

The method described above was translated into a FORTRAN 90 program and was run on a pentium IV personal computer. The value of $\zeta_{\infty}$, the numerical infinity has been taken large enough and kept invariant through out the run of the program. The value of $\zeta_{\infty}=10.0$ is found to be adequate for all the 
cases shown in Figs. 2-25, however, for higher values of the non-Newtonian flow parameters, the numerical integrations are performed over substantially larger domain to ensure that the outer boundary conditions at $\zeta=\infty$ are satisfied. To see if the program runs correctly, the value of the missing initial condition $-\varphi^{\prime \prime}(0)$ for Newtonian $(K \rightarrow 0, L \rightarrow 0, \beta \rightarrow 0)$ and non-magnetic flow $\left(M_{n} \rightarrow 0\right)$ is compared with the values reported by Elbashbeshy [11] and Magyari and Keller [21] in their pioneer works. We found $-\varphi^{\prime \prime}(0)=1.281811$ correct to six decimal places after the seventh iteration, which is in good agreement with $-f^{\prime \prime}(0)=1.28181$ and $-f^{\prime \prime}(0)=1.28180$ reported in [11] and [21] respectively.

Figs. 2-6 elucidate the effects of the different flow parameters on $\varphi(\zeta)$, which represents the normalized downward vertical velocity. It is interesting to find from Fig. 2 that even the presence of small amount of slip $(\lambda=0.1)$ dominates the conventional effects of the viscoelasticity $(K)$ on $\varphi(\zeta)$, i.e. in presence of slip, $\varphi(\zeta)$ decreases with an increase in $K$. This finding can be regarded as a step forward towards answering the still unresolved issue of what would be the effects of a fluid's elasticity on the characteristics of its boundary layer in presence of slip. The vertical component of velocity also decreases with an increase in the cross-viscous parameter $L$, as is clear from Fig. 3. The effects of the shear thickening parameter $\beta$ and the magnetic parameter $M_{n}$ on $\varphi(\zeta)$ have been shown in Figs. 4 and 5 respectively. Fig. 6 shows that for a given position $\zeta$, as the slip increases, $\varphi(\zeta)$ decreases.

Figs. 7-11 indicate the variations of the horizontal velocity similarity profile $\varphi^{\prime}(\zeta)$ with different flow parameters. The dominating nature of slip on the viscoelasticity is again clear from Fig. 7 . One can see that in presence of slip, as $K$ increases, $\varphi^{\prime}(\zeta)$ decreases near the surface of the sheet, and then increases away from it resulting a 'cross over' in the velocity profile. We found that with an increase in the slip factor $\lambda$, the position of the cross over shifts towards the $\zeta$-axis, and eventually coincides with it, resulting a decrease of $\varphi^{\prime}(\zeta)$ with an increase in $K$, throughout the domain of integration. Fig. 8 depicts that $\varphi^{\prime}(\zeta)$ decreases with $L$. Thus, the cross-viscous parameter decreases the momentum boundary layer thickness. The third grade fluid parameter $\beta$, on the other hand has an opposite effect on $\varphi^{\prime}(\zeta)$, as is clear from Fig. 9. Fig. 10 shows the pronounced effect of the magnetic field on $\varphi^{\prime}(\zeta)$. The velocity decreases with an increase in the magnetic parameter $M_{n}$. That is the Lorentz force which opposes the flow leads to enhanced deceleration of the flow. In Fig. 11 the main stream velocity $\varphi^{\prime}(\zeta)$ has been plotted against $\zeta$ for different values of the slip parameter $\lambda$. It can be readily observed that as the slip parameter increases in magnitude, permitting more fluid to slip past the sheet, the flow slows down for distances close to the sheet. The effect of slip, away from the sheet is less pronounced. Due to slip, the velocity on the boundary $\varphi^{\prime}(0)$ remains less than the normalized stretching surface velocity of unity. 
The effects of various flow parameters on the non-dimensional temperature profiles $\theta(\zeta)$ for the PST case have been delineated in Figs. 12-16 for $P_{r}=5.0$ and $E_{c}=0.5$. It is clear that the effect of $\beta$ is to reduce the thermal boundary layer thickness. On the other hand, an increase in slip increases the thermal boundary layer thickness. The variations of the non-dimensional temperature profiles $g(\zeta)$ for the PHF case with different flow parameters are shown in Figs. 17-21 for $P_{r}=5.0$ and $E_{c}=0.5$. It is found that the respective flow parameters have similar effects on $\theta(\zeta)$ and $g(\zeta)$.

The local skin-friction coefficient or frictional drag coefficient is given by

$$
C_{f}(x)=\frac{\left.T_{x y}\right|_{y=0}}{\frac{1}{2} \rho U_{w}^{2}}
$$

which in terms of the dimensionless quantities is

$$
C_{f}(x)=\sqrt{2} R_{l}^{-\frac{1}{2}}\left[\varphi^{\prime \prime}+K\left(\frac{7}{2} \varphi^{\prime} \varphi^{\prime \prime}-\frac{1}{2} \varphi \varphi^{\prime \prime \prime}\right)+\beta \varphi^{\prime \prime 3}\right]_{\zeta=0} .
$$

Here, $R_{l}=\frac{U_{w} l}{\nu}$ represents the local Reynolds number. Figs. 22-25 elucidate the variations of the skin-friction coefficient $C_{f}(x)$ with different flow parameters in presence of slip with $R_{l}=1.0$. Fig. 22 shows that the skin-friction coefficient is increased in magnitude with an increase in the viscoelasticity of the fluid. This is in agreement with our previous study [5] on the flow and heat transfer of a third grade fluid past a planar stretching sheet subject to partial slip boundary conditions. This prediction is of course undesirable from an industrial standpoint, because it translates into a larger driving force (or torque). In a subsequent figure (see Fig. 23), we have shown the variation of $C_{f}(x)$ with the cross-viscous parameter $L$. It is interesting to find a turning point in the $C_{f}(x)$ profile. In fact, $C_{f}(x)$ increases in magnitude till certain value of $L$ (critical value not precisely determined), and then starts falling rapidly with an increase in $L$. The shear thickening parameter $\beta$ decreases the magnitude of $C_{f}(x)$, as is clear from Fig. 24. Finally, we plot the effects of slip on $C_{f}(x)$ in Fig. 25. It is apparent that the skin-friction coefficient decreases rapidly, and approaches zero as the slip starts increasing. A precise idea regarding the variations of the dimensionless surface temperature gradient $\theta^{\prime}(0)$ in the PST case and the dimensionless surface temperature $g(0)$ in the PHF case with emerging flow parameters can be obtained from Table 1. 


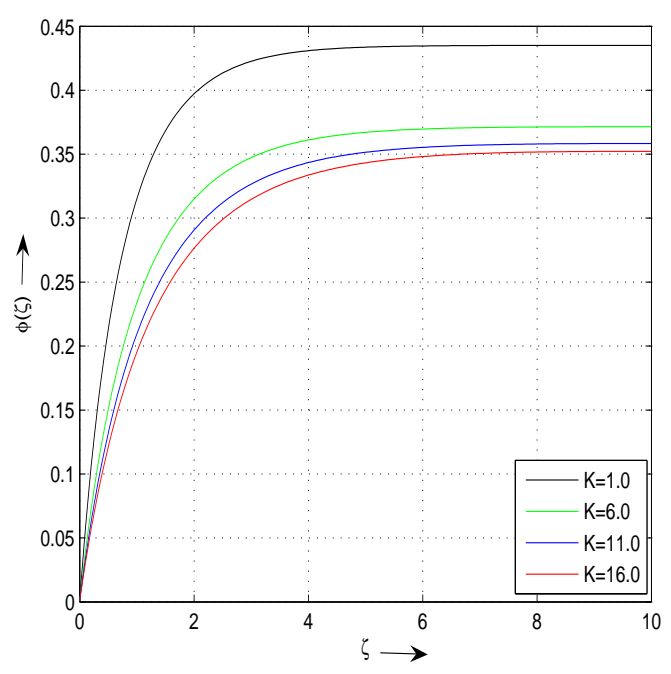

Fig. 2. Variation of $\varphi$ with $K$ at $L=1.0, \beta=1.0, M_{n}=0.5 \&$ $\lambda=0.1$.

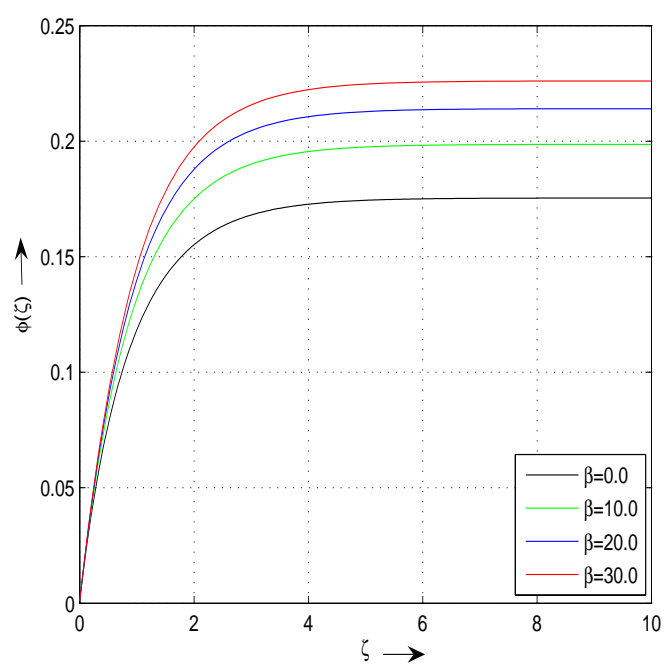

Fig. 4. Variation of $\varphi$ with $\beta$ at $K=2.0, L=1.0, M_{n}=0.5 \&$ $\lambda=1.0$.

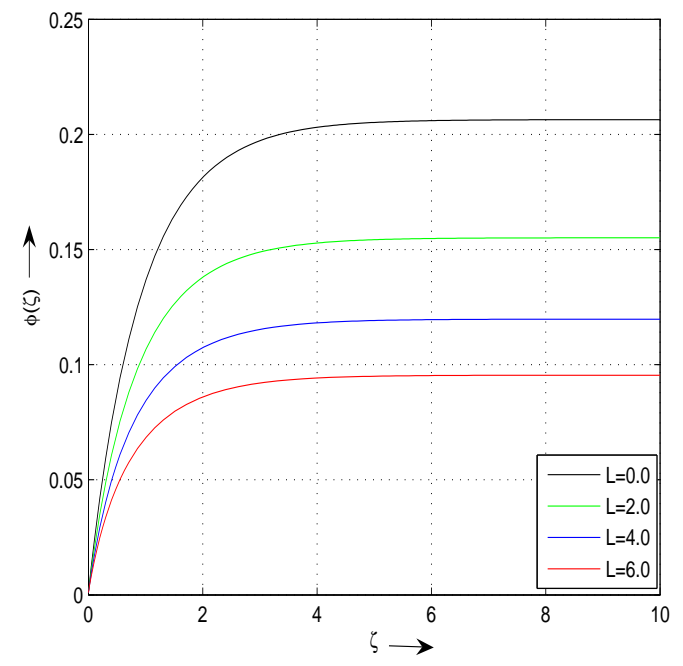

Fig. 3. Variation of $\varphi$ with $L$ at $K=2.0, \beta=1.0, M_{n}=0.5 \&$ $\lambda=1.0$.

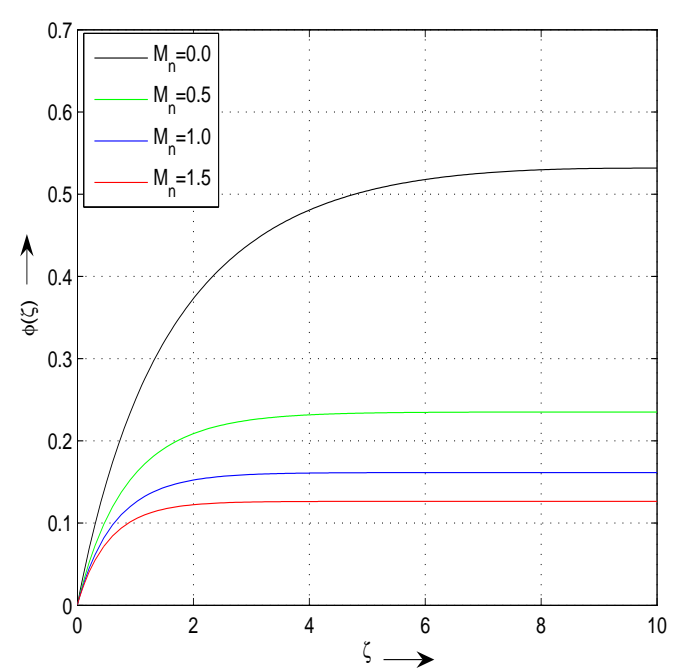

Fig. 5. Variation of $\varphi$ with $M_{n}$ at $K=2.0, L=1.0 \& \beta=1.0 \&$ $\lambda=0.5$. 


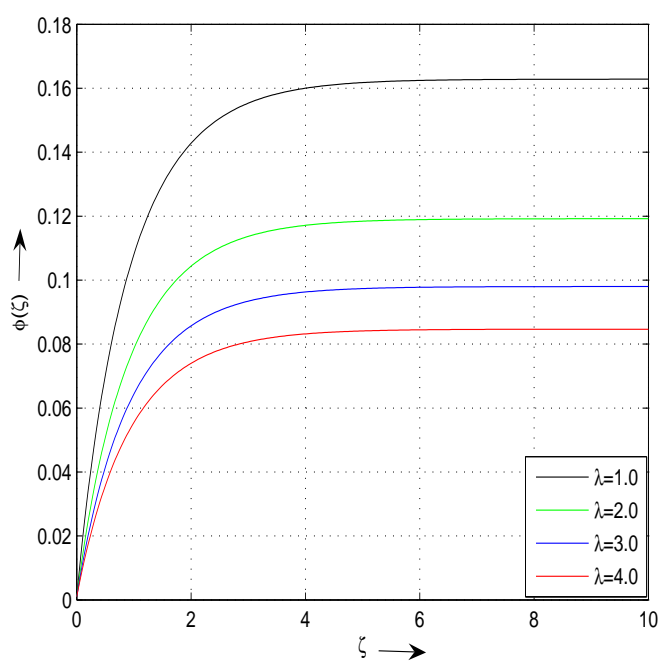

Fig. 6. Variation of $\varphi$ with $\lambda$ at $K=3.0, L=1.0, \beta=1.0$, $M_{n}=0.5$.

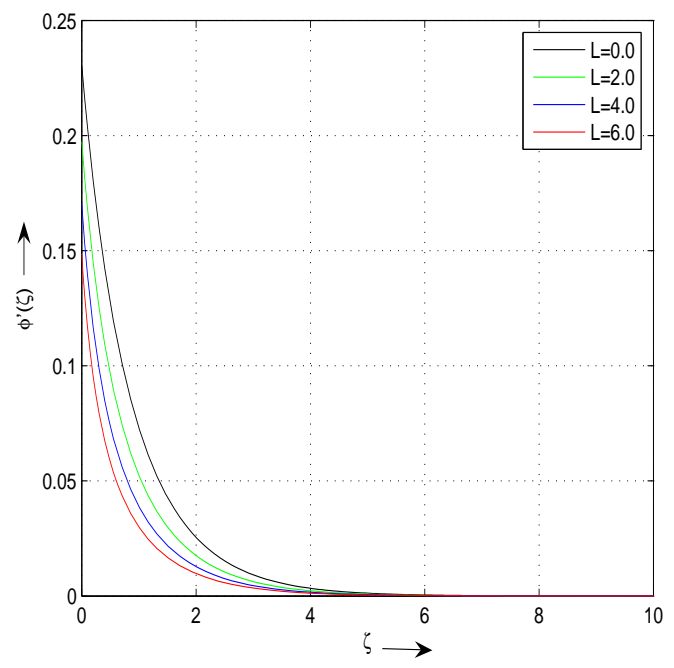

Fig. 8. Variation of $\varphi^{\prime}$ with $L$ at $K=2.0, \beta=1.0, M_{n}=0.5 \&$ $\lambda=1.0$.

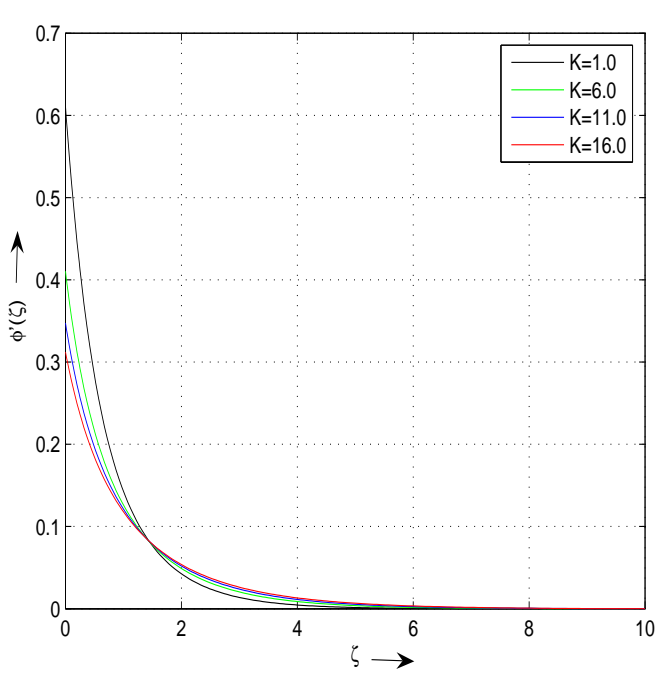

Fig. 7. Variation of $\varphi^{\prime}$ with $K$ at $L=1.0, \beta=1.0, M_{n}=0.5 \&$ $\lambda=0.1$.

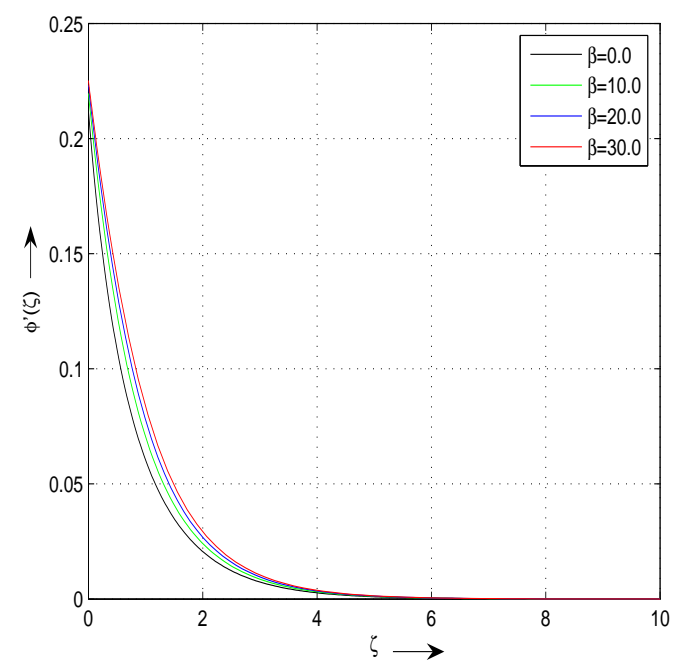

Fig. 9. Variation of $\varphi^{\prime}$ with $\beta$ at $K=2.0, L=1.0, M_{n}=0.5 \&$ $\lambda=1.0$. 


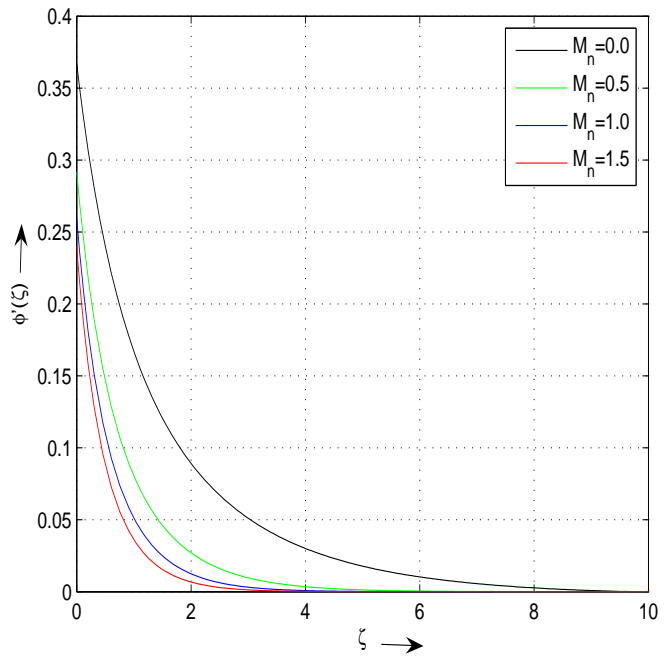

Fig. 10. Variation of $\varphi^{\prime}$ with $M_{n}$ at $K=2.0, L=1.0, \beta=1.0 \&$ $\lambda=0.5$.

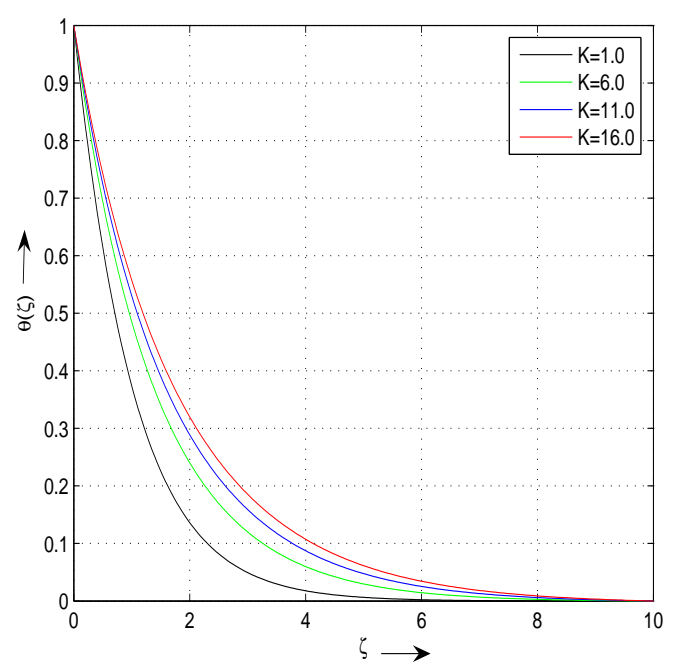

Fig. 12. Variation of $\theta$ with $K$ at $L=1.0, \beta=1.0, M_{n}=0.5$, $\lambda=1.0$.

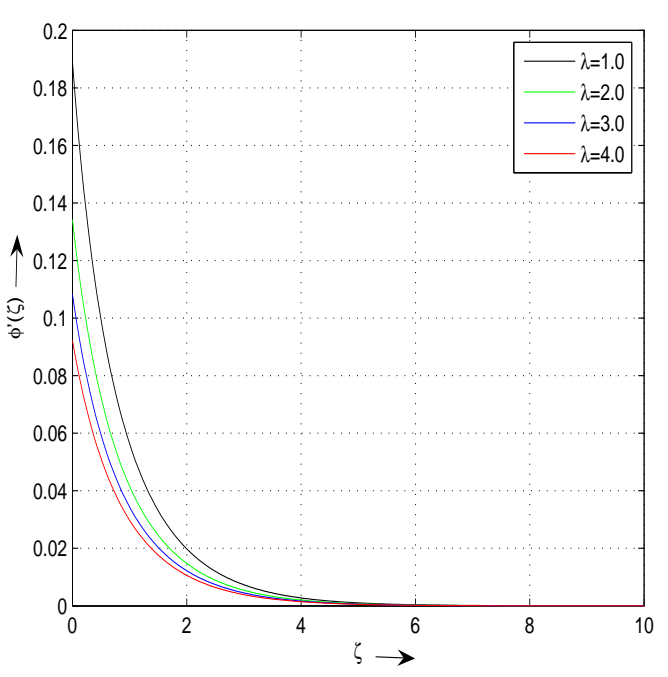

Fig. 11. Variation of $\varphi^{\prime}$ with $\lambda$ at $K=3.0, L=1.0, \beta=1.0$, $M_{n}=0.5$.

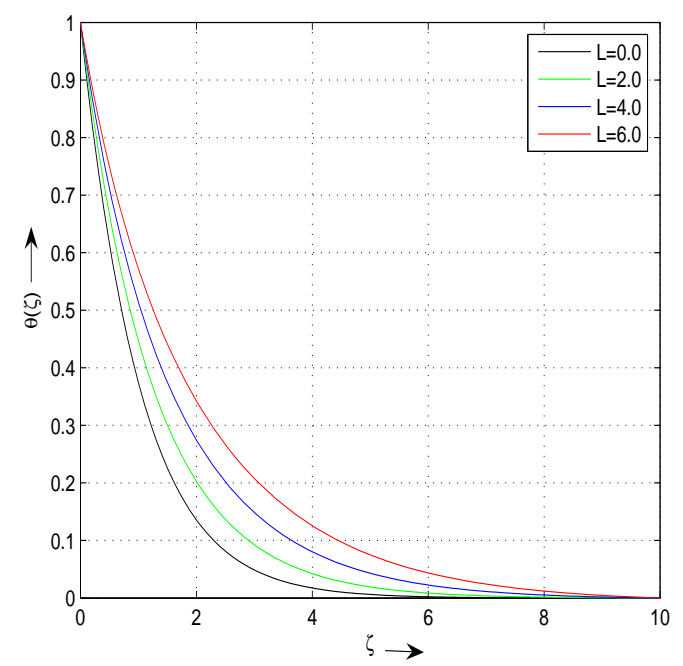

Fig. 13. Variation of $\theta$ with $L$ at $K=2.0, \beta=1.0, M_{n}=0.5$, $\lambda=1.0$. 


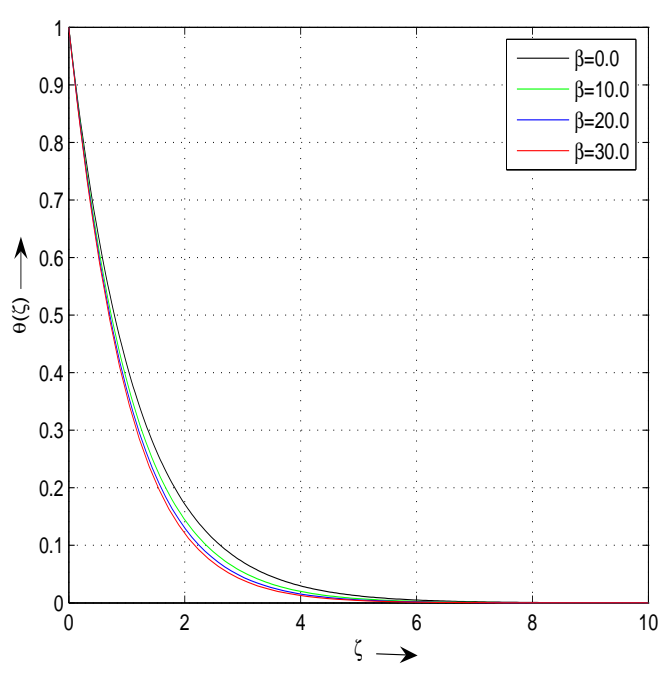

Fig. 14. Variation of $\theta$ with $\beta$ at $K=2.0, L=1.0, M_{n}=0.5$, $\lambda=1.0$.

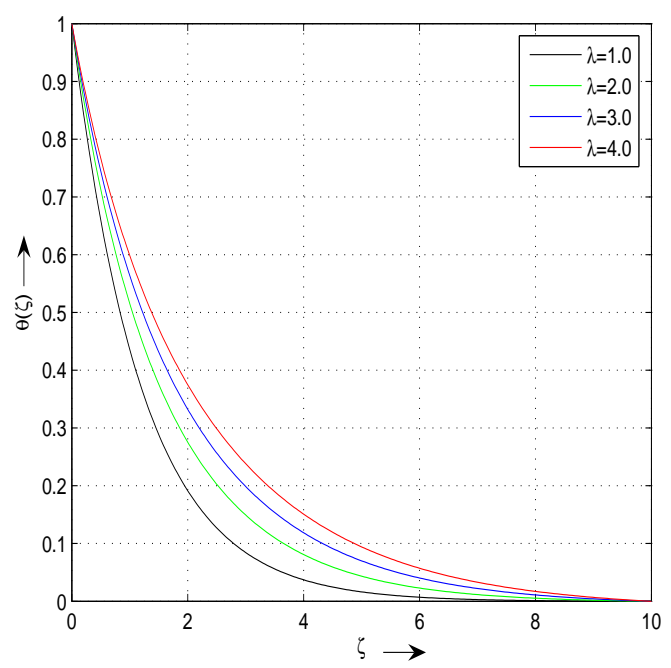

Fig. 16. Variation of $\theta$ with $\lambda$ at $K=3.0, L=1.0, \beta=1.0$, $M_{n}=0.5$.

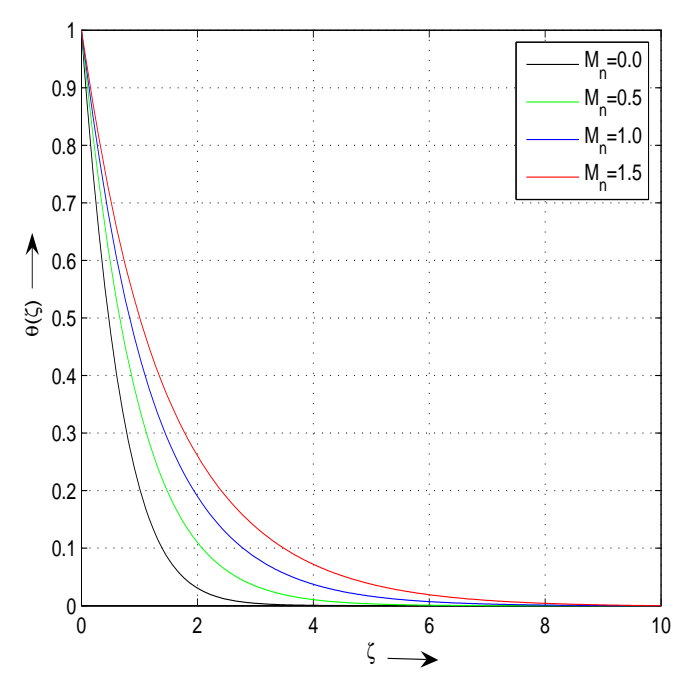

Fig. 15. Variation of $\theta$ with $M_{n}$ at $K=2.0, L=1.0, \beta=1.0, \lambda=0.5$.

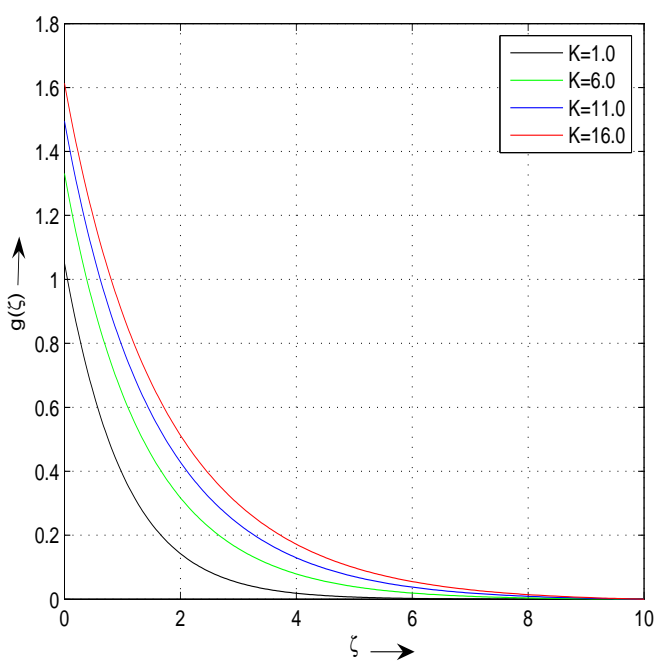

Fig. 17. Variation of $g$ with $K$ at $L=1.0, \beta=1.0, M_{n}=0.5$, $\lambda=1.0$. 


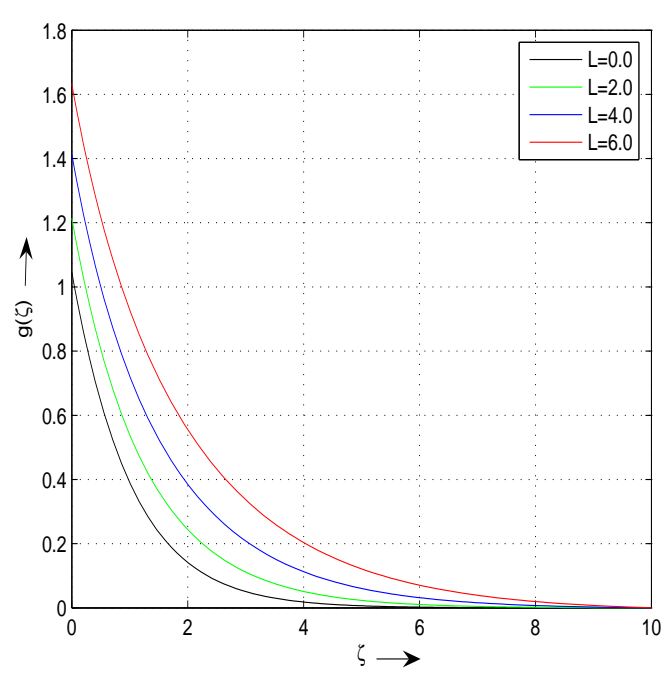

Fig. 18. Variation of $g$ with $L$ at $K=2.0, \beta=1.0, M_{n}=0.5$, $\lambda=1.0$.

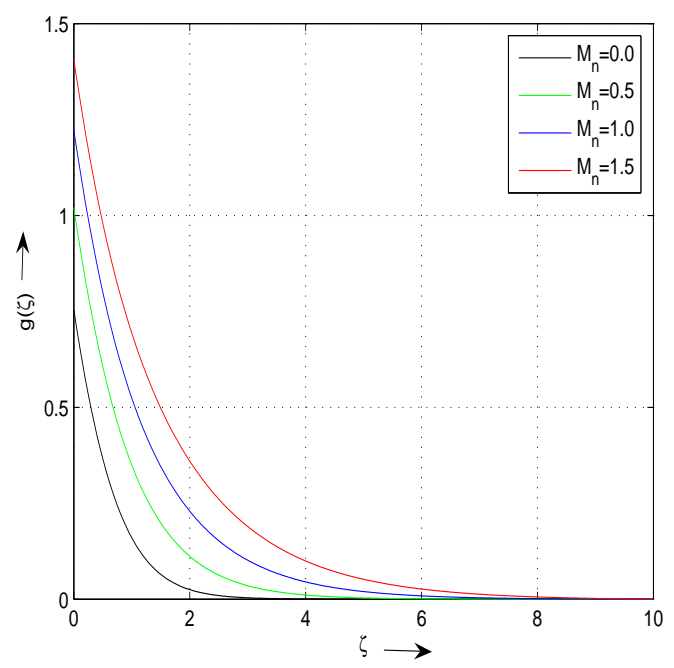

Fig. 20. Variation of $g$ with $M_{n}$ at $K=2.0, L=1.0 \& \beta=1.0$, $\lambda=0.5$.

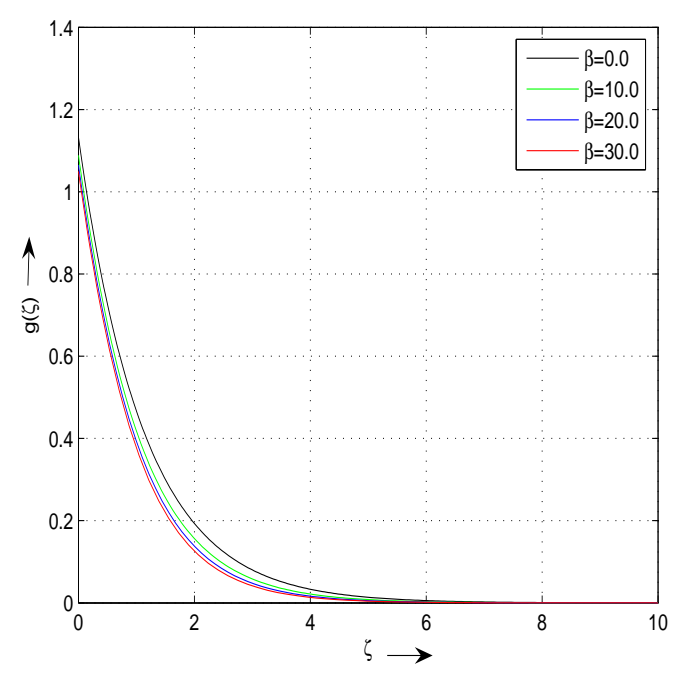

Fig. 19. Variation of $g$ with $\beta$ at $K=2.0, L=1.0, M_{n}=0.5$, $\lambda=1.0$.

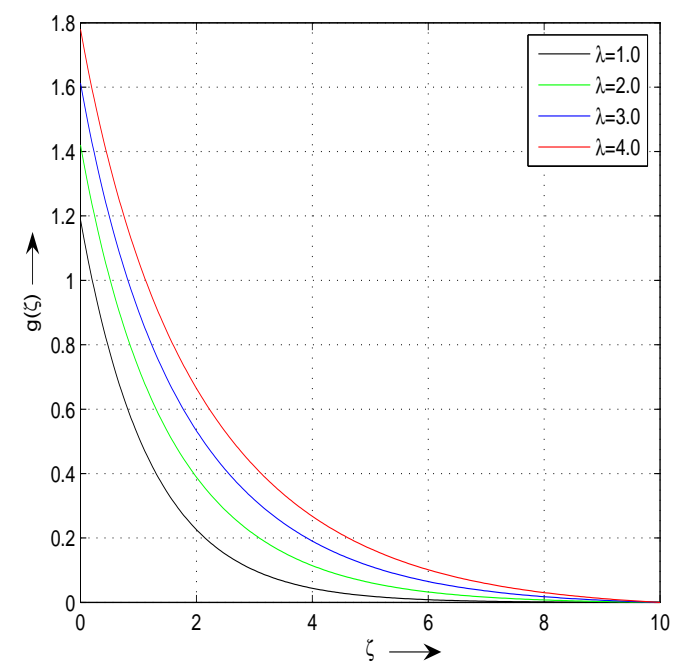

Fig. 21. Variation of $g$ with $\lambda$ at $K=3.0, L=1.0, \beta=1.0$, $M_{n}=0.5$. 


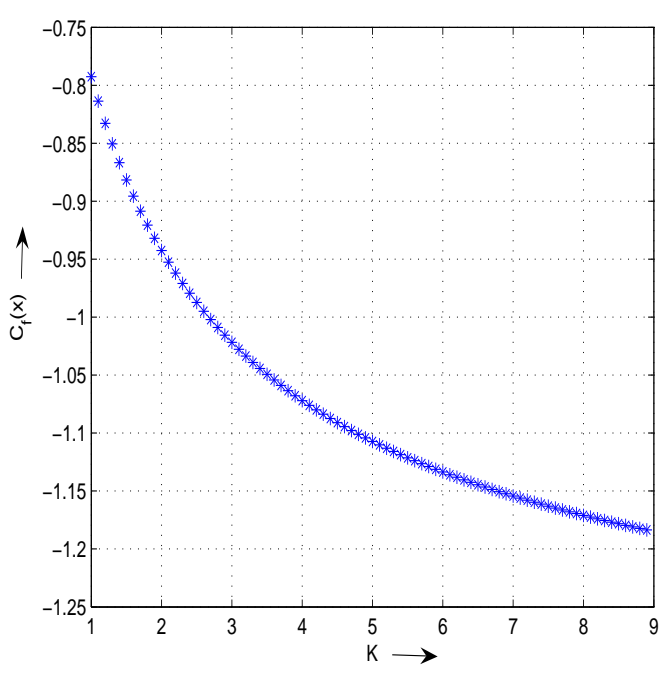

Fig. 22. Variation of $C_{f}(x)$ with $K$ at $L=1.0, \beta=1.0, M_{n}=0.5 \&$ $\lambda=1.0$.

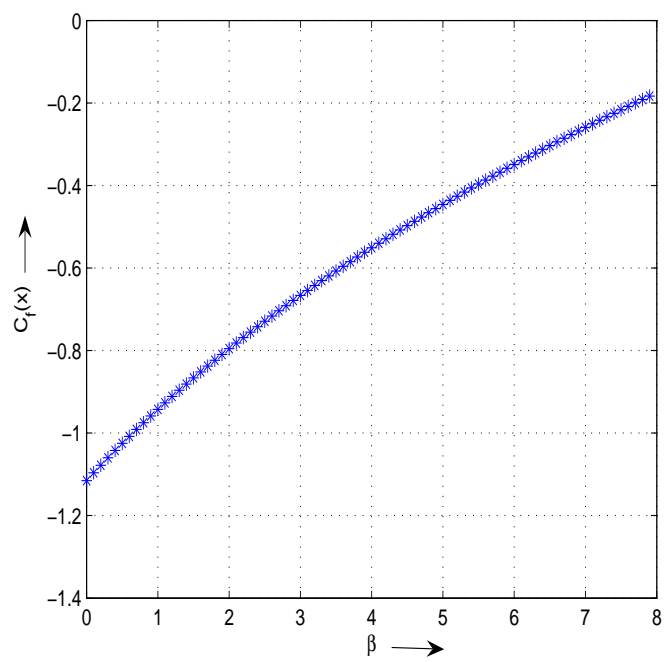

Fig. 24. Variation of $C_{f}(x)$ with $\beta$ at $K=2.0, L=1.0, M_{n}=0.5 \&$ $\lambda=1.0$.

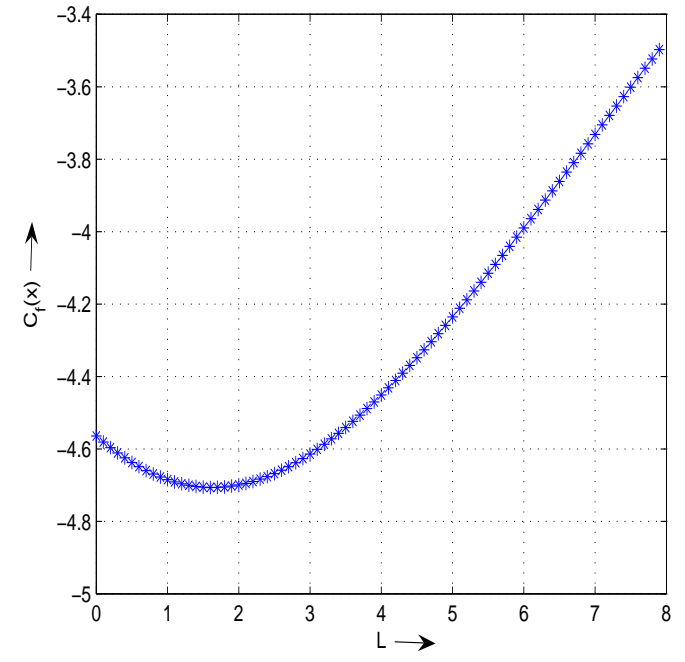

Fig. 23. Variation of $C_{f}(x)$ with $L$ at $K=2.0, \beta=1.0, M_{n}=0.5 \&$ $\lambda=0.1$

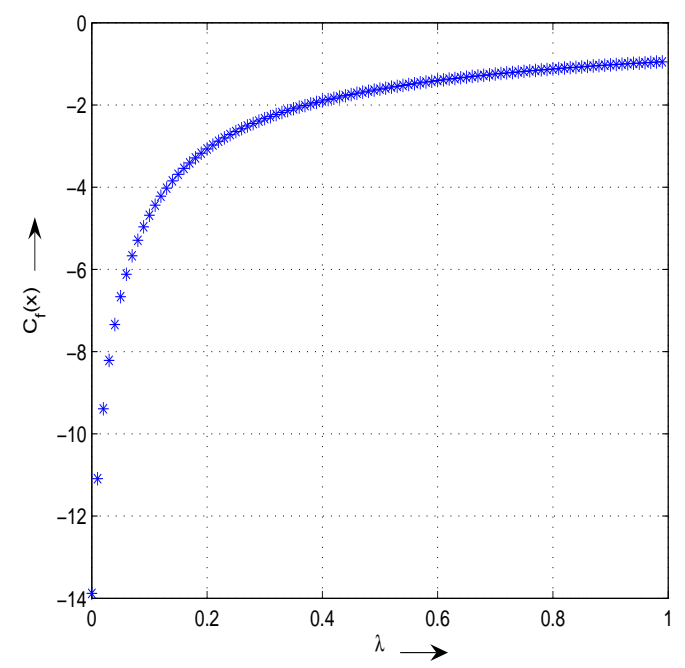

Fig. 25. Variation of $C_{f}(x)$ with $\lambda$ at $K=2.0, L=1.0, \beta=1.0 \&$ $M_{n}=0.5$. 
Table 1

Variations of $\theta^{\prime}(0)$ and $g(0)$ with different flow parameters at $P_{r}=3.0$ and $E_{c}=0.5$.

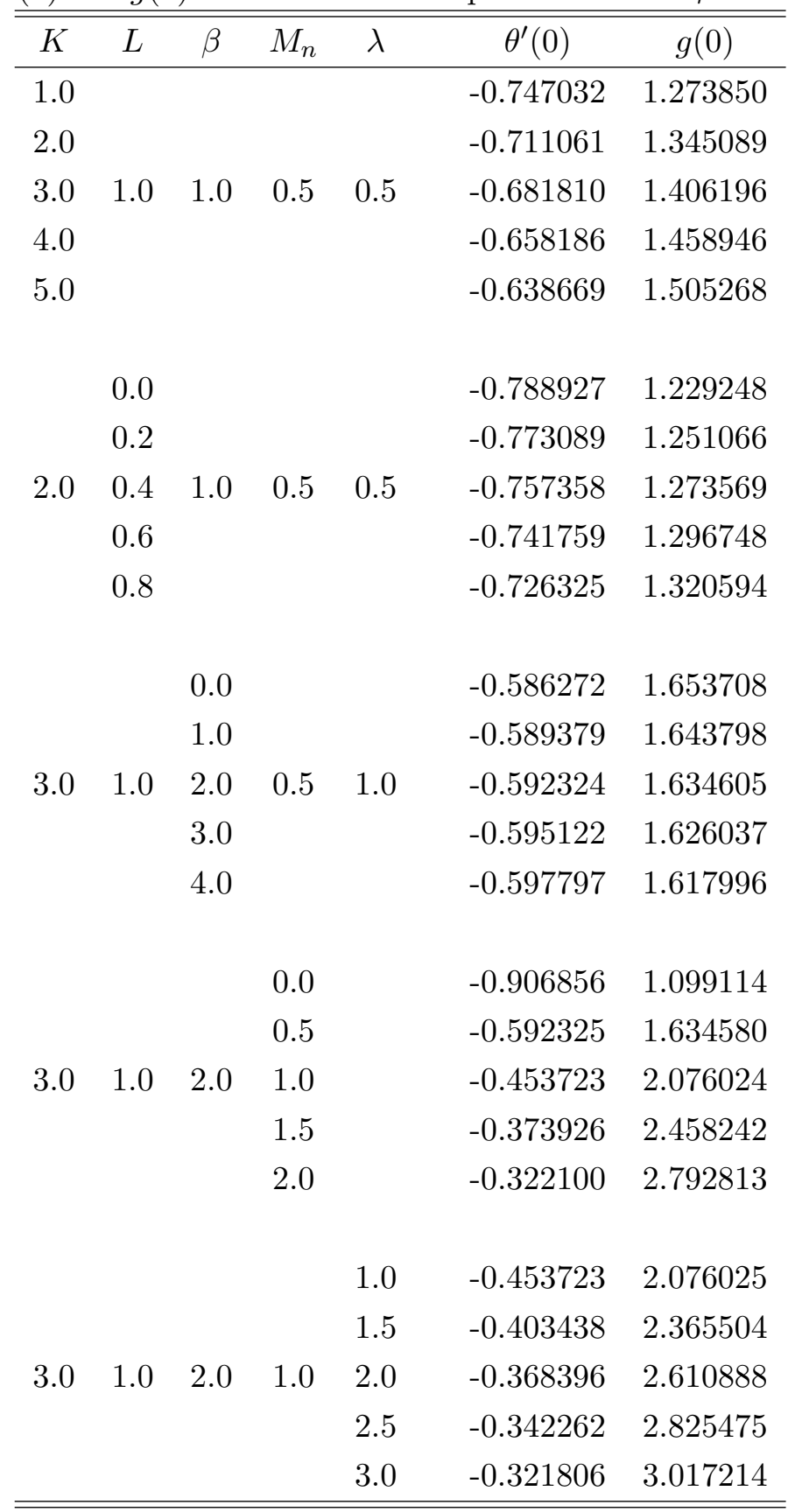

\section{Conclusions}

The present work deals with the numerical investigation of the effects of partial slip on the steady flow and heat transfer of an electrically conducting third grade fluid due an exponentially stretching sheet. The heat transfer analysis has been carried out for two heating processes, namely (i) with prescribed surface temperature (PST), and (ii) prescribed heat flux (PHF). It is found 
that the third grade fluid parameter $\beta$ increases the momentum boundary layer thickness and decreases the thermal boundary layer thickness. The nondimensional slip factor $\lambda$ has substantial effect on the momentum as well as on the thermal boundary layers. The amount of slip $1-\varphi^{\prime}(0)$ increases monotonically with $\lambda$ from the no-slip solution $\lambda=0$ to the full-slip as $\lambda$ tends to infinity. It is interesting to find a turning point in the curve $\left(C_{f}(x), L\right)$. Moreover, it is found that the skin-friction coefficient $C_{f}(x)$ decreases exponentially as the slip starts increasing from zero. It is also found that the shear thickening parameter $\beta$ increases the magnitude of the rate of heat transfer $\theta^{\prime}(0)$ at the surface of the sheet, whereas, the slip factor has an opposite effect on it. The obtained solutions have promising applications in engineering and can be regarded as a step forward towards answering the still unresolved issue pertaining to the boundary layer flows of third grade fluid subject to partial slip boundary conditions.

\section{References}

[1] C.L.M.H. Navier. Sur les lois du mouvement des fluides. Mem. Acad. R. Acad. R. Sci. Inst. Fr., 6:389-440, 1827.

[2] H.I. Andersson. Slip flow past a stretching surface. Acta Mech., 158:121-125, 2002.

[3] B. Sahoo. Flow and heat transfer of a non-Newtonian fluid past a stretching sheet with partial slip. Comm. Nonlin. Sci. Num. Sim., 15:602-615, 2010.

[4] B. Sahoo. Effects of partial slip on axisymmetric flow of an electrically conducting viscoelastic fluid past a stretching sheet. Cent. Euro. Jr. Phy., 8:498-508, 2010.

[5] B. Sahoo and Y. Do. Effects of slip on sheet-driven flow and heat transfer of a third grade fluid past a stretching sheet. Int. Comm. Heat Mass Trans., $37: 1064-1071,2010$.

[6] P.D. Ariel. Axisymmetric flow due to a stretching sheet with partial slip. Int. Jr. Comp. Maths.

[7] M. Sajid, T. Hayat, and S. Asghar. Non-similar analytic solution for MHD flow and heat transfer in a third-order fluid over a stretching sheet. Int. Jr. Heat and Mass Trans., 50:1723-1736, 2007.

[8] R. Ellahi, T. Hayat, and S. Asghar. Effects of slip on non-linear flows of a third grade fluid. Nonl Anal: Real World Appl., 11:139-146, 2010.

[9] S. Ashgar, C. Khalque, and R. Ellahi. Influence of partial slip on flows of second grade fluid in a porous medium. Jr. Porous Media, DOI:10.1615/JPorMedia.v10.i8.50, pages 797-805, 2010. 
[10] S. Nadeem, T. Hayat, S. Abbasbandy, and M. Ali. Effects of partial slip on a fourth grade fluid with variable viscosity: An ananytic solution. Nonl Anal: Real World Appl., 11:856-868, 2010.

[11] E.M.A. Elbashbeshy. Heat transfer over an exponentially stretching continuous surface with suction. Arch. Mech., 53:643-651, 2001.

[12] M.K. Parhta, PVSN Murthy, and G.P. Rajasekhar. Effects of viscous dissipation on the mixed convection heat transfer from an exponentially stretching surface. Heat Mass Trans., 41:360-366, 2005.

[13] M. Sajid and T. Hayat. Influence of thermal radiation on the boundary layer flow due to an exponentially stretching sheet. Int. Jr. Heat and Mass Trans., 35:347-356, 2008.

[14] D. Pal. Mixed convection heat transfer in the boundary layers on an exponentially stretching surface with magnetic field. Appl. Math. Comp. doi: 10.1016/j.amc.2010.07.035, 2010.

[15] M.Q. Al-Odat, R.A. Damseh, and T.A. Al-Azab. Thermal boundary layer on an exponentially stretching continous surface in the presence of magnetic field effect. Int. J. of Appl Mech and Engng, 11:289-299, 2006.

[16] B. Bidin and R. Nazar. Numerical solution of the boundary layer flow over an exponentially stretching sheet with thermal radiation. Eur. Jr. of Sci. Res., 33:710-717, 2009.

[17] S.K. Khan and E. Sanjayanand. Viscoelastic boundary layer flow and heat transfer over an exponential stretching sheet. Int. Jr. Heat Mass Trans., 48:1534-1542, 2005.

[18] S.K. Khan. Boundary layer viscoelastic fluid flow over an exponentially stretching sheet. Int. Jr. Appl. Mech. and Engng., 11:321-335, 2006.

[19] E. Sanjayanand and S.K. Khan. On heat and mass transfer in a viscoelastic boundary layer flow over an exponentially stretching sheet. Int. Jr. Thermal Sci., 45:819-828, 2006.

[20] J.E. Dunn and K.R. Rajagopal. Fluids of differential type: Critical review and thermodynamic analysis. Int. Jr. Engng. Sci., 33:689-729, 1995.

[21] E. Magyari and B. Keller. Heat and mass transfer in the boundary layers on an exponentially stretching continuous surface. J. Phys. D: Appl.Phys., 32:577585,1999 .

[22] M. Pakdemirli. The boundary layer equations of third grade fluids. Int. Jr. Non-Linear Mech., 27:785-793, 1992.

[23] C.G. Broyden. A class of methods for solving nonlinear simultaneous equations. Math. Comp, 19:577-93, 1965.

[24] C.G. Broyden. On the discovery of the good broyden method. Math Program Ser B, 87:209-213, 2000. 